\title{
Mitteilungen aus der AO
}

\author{
George Clay
}

\section{Ausschreibung \\ AOTD Nachwuchsförderung}

Die AOTrauma Deutschland (AOTD) fördert den wissenschaftlichen Nachwuchs im klinischen und experimentellen Bereich mit einer Anschubfinanzierung sowie einem Mentoring. Anträge können bis Montag, 31. August 2020 eingereicht werden.

Bewerben können sich junge (Facharzt/ärztin plus 2 Jahre), klinisch aktive Unfallchirurginnen/en, die in Deutschland an Universitätskliniken sowie regionalen und kommunalen Krankenhäusern tätig sind. Funktion Oberarzt/ärztin oder Leitende/r OA sind keine Ausschlusskriterien. Habilitierte dürfen sich nicht bewerben.
Eingereicht werden können sowohl klinische als auch experimentelle Forschungskonzepte aus den Bereichen Traumatologie, Mund-Kiefer-Gesichts-Chirurgie und Veterinärchirurgie (nicht Wirbelsäule). Der/die Antragsteller/in muss ein/-e Kliniker/-in sein. Es werden nur Bewerbungen von Mitgliedern der deutschen AOTrauma, AOCMF und AOVET angenommen, deren Forschungsarbeit von der jeweiligen Klinikleitung unterstützt wird.

Aus den eingegangenen Bewerbungen wählt die AOTD 4 Beiträge aus und lädt die Antragsteller/innen ein, ihre Arbeiten auf der Auswahlsitzung während des DKOU im Oktober 2020 in Berlin vorzustellen und zu verteidigen.

Zwei der Kandidatinnen/en werden dann mit einer Anschubfinanzierung von maximal 15000 Euro ausgezeichnet. Die Anwesenheit in Berlin ist Voraussetzung zur Teilnahme.

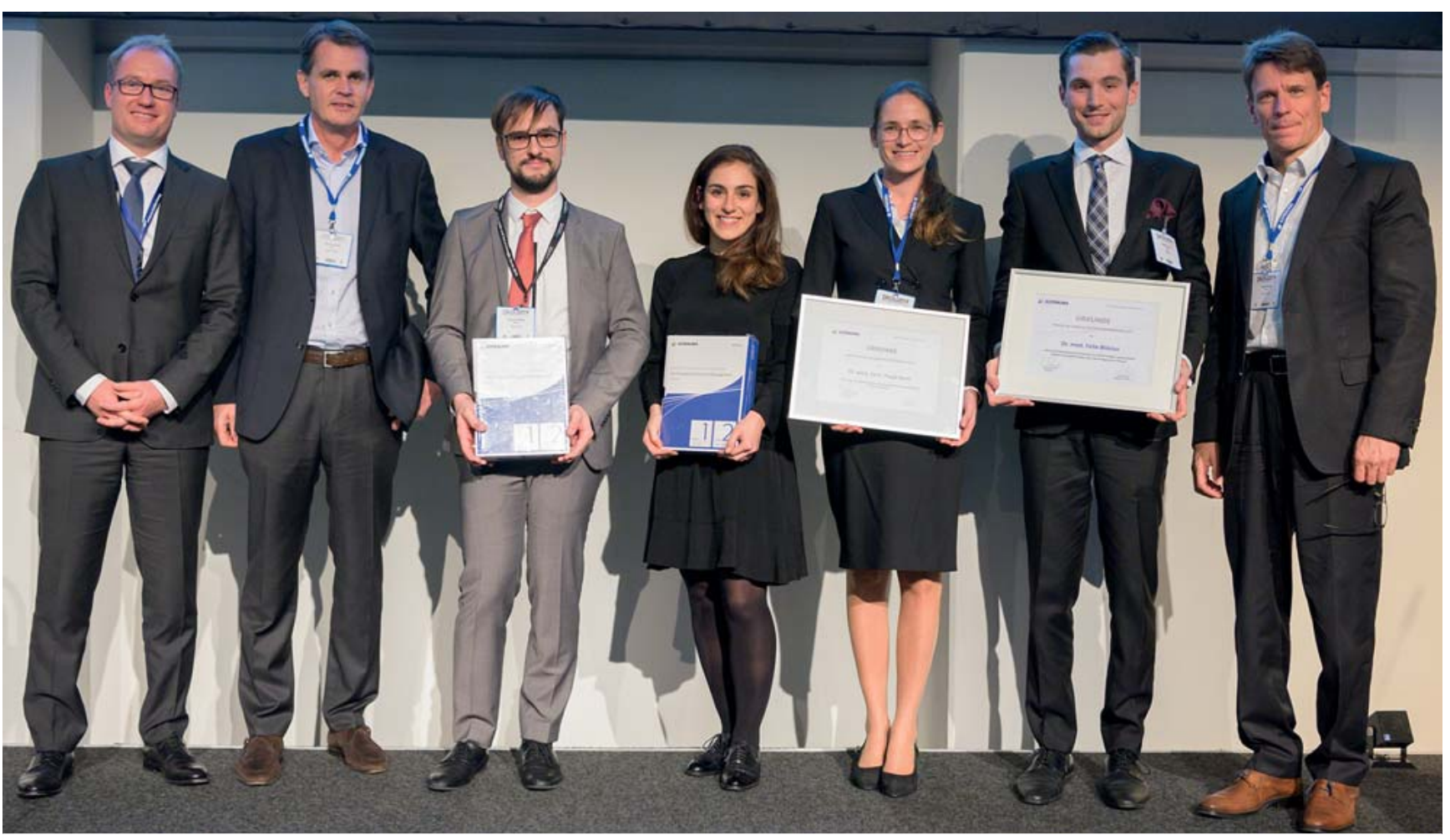

- Abb. 1 Teilnehmende der Preisträgersitzung im Oktober 2019 in Berlin auf der DKOU. Von links nach rechts: Richard Stange, Ulrich Stöckle, Philipp Pieroh, Lisa Oezel, Paula Korn, Felix Bläsius, Georg Duda. Quelle: AO Foundation 


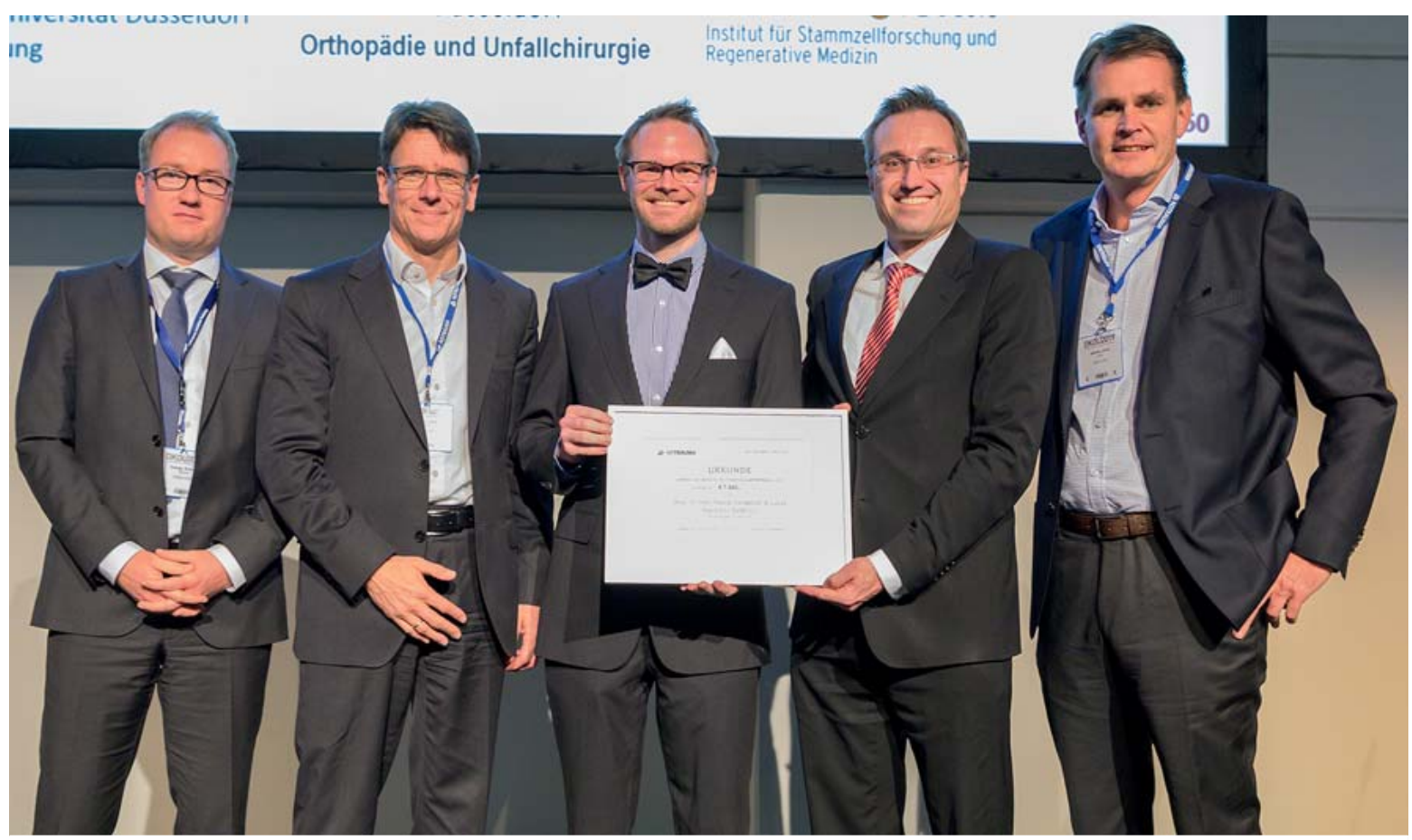

- Abb. 2 Gewinner waren zusammen Lucas Sebastian Spitzhorn und Pascal Jungbluth. Von links nach rechts: Richard Stange, Georg Duda, Lucas Sebastian Spitzhorn, Pascal Jungbluth, Ulrich Stöckle. Quelle: AOTD

Bitte beachten Sie, dass

- pro Ausschreibung darf nur ein Antrag pro Bewerber/ in eingereicht werden,

- nach Ablehnung eines Antrages ist nur eine Wiedereinreichung desselben Antrages (mit der gleichen Thematik) möglich,

- wenn ein/e Bewerber/in schon einmal gefördert wurde, darf er/sie kein zweites Mal ein Projekt einreichen, auch kein anderes,

- bei einer Ausschreibung dürfen mehrere Anträge aus derselben Klinik eingereicht werden, aber pro Klinik wird nur ein Antrag zur Preisträgersitzung zugelassen.

Das Antragsformular für Nachwuchsförderung finden Sie auf der AOTD-Homepage (www.aotrauma.org/deutschland); es ist ferner über die Geschäftsstelle (g.clay@ aodeutschland.org) der AOTD zu erhalten.

Die Anträge sind bis zum 31. August 2020 zu senden an: Prof. Dr. Georg Duda

Research Officer der AOTrauma Deutschland

E-Mail: nachwuchsfoerderung@aodeutschland.de

\section{AOTrauma Deutschland}

\section{Wissenschaftspreis 2020}

Für den Wissenschaftspreis 2020 der AOTrauma Deutschland werden noch Bewerbungen bis zum Montag, 31.08.2020 entgegengenommen. Der Preis wird im Ok- tober 2020 auf einer AOTrauma Deutschland-Sitzung im Rahmen des DKOU in Berlin vergeben, auf dem die Arbeit präsentiert werden darf. Das Preisgeld beträgt 7500 EUR.

Voraussetzungen sind:

- Die Arbeit muss bereits in einem Peer-reviewed Journal publiziert sein.

- Das Thema der Arbeit muss im Tätigkeitsbereich der AOTrauma, AOCMF oder AOVET liegen.

- Es können sowohl klinische Forschungsarbeiten, Grundlagenforschung, systematische Reviews als auch Metaanalysen eingereicht werden.

- AO-Mitgliedschaft (Trauma, CMF oder VET)

- Anwesenheit auf der Sitzung während des DKOU im Oktober 2020 in Berlin

Einzureichen sind bis zum 31. August 2020 die Arbeit, ein Motivationsbrief und der Lebenslauf an Prof. Dr. Georg Duda, Research Officer der AOTrauma Deutschland, E-Mail: wissenschaftspreis@aodeutschland.de

Mehr Informationen finden Sie auf der Homepage der AOTD in der Rubrik unter www.aotrauma.org/deutschland 


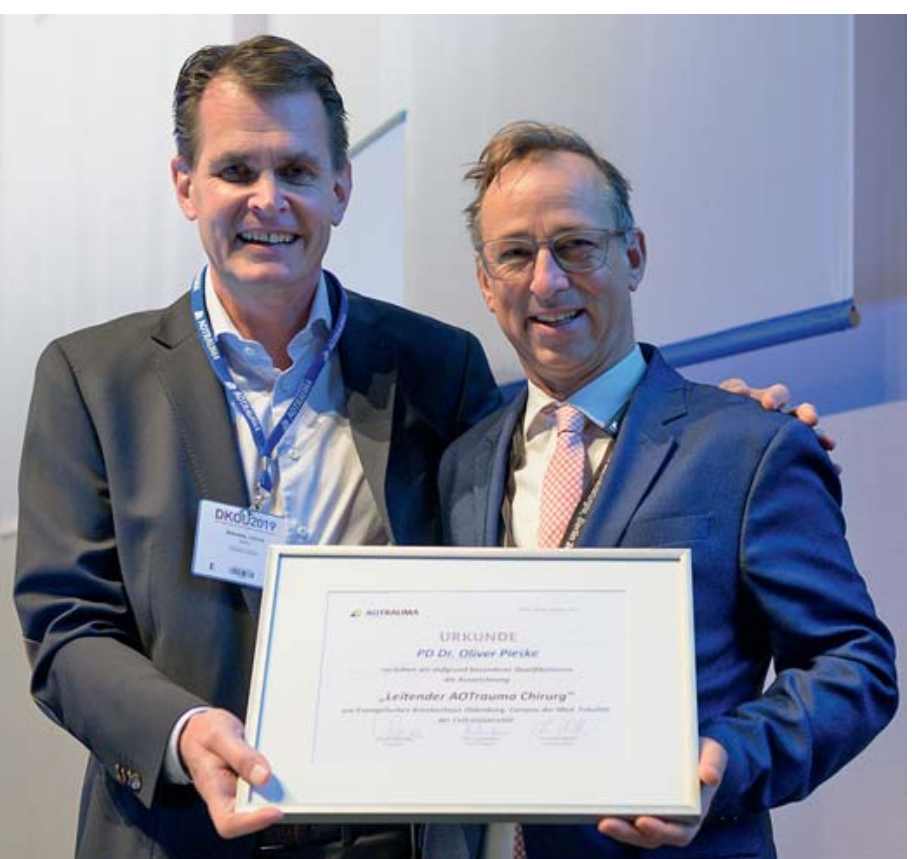

- Abb. 3 Bei der Auszeichnung in Berlin auf der DKOU. Von links nach rechts: Ulrich Stöckle, Oliver Pieske. Quelle: AOTD

\section{Ausschreibung für eine Auszeichnung "Leitende/r AOTrauma Chirurg/in“"}

\section{Ziel der Auszeichnung}

Mit dieser Auszeichnung möchte die AOTD die Sichtbarkeit der ausgezeichneten Person sowie die Qualität und Attraktivität der Einrichtung erhöhen, an der sie tätig ist. Weiterhin soll ein zusätzlicher Anreiz für die Weiter- bildung der Mitarbeiter/innen geschaffen werden, insbesondere hinsichtlich der Angebote der AO, um die AOPrinzipien zu lernen, kontinuierlich zu vertiefen und zu verbreiten. Insofern dient die Auszeichnung einerseits der Darstellung der Exzellenz von Mitarbeiter und Klinik anhand von transparenten Kriterien, andererseits dem Ausbau der „AO-Schule“. Es handelt sich hierbei nicht um eine personelle oder gar institutionelle Zertifizierung, sondern um eine persönliche Auszeichnung ausschließlich für Mitglieder der AOTD.

\section{Auswahlkriterien und Deadline für nächste Antragsrunde}

Zur Verleihung der Auszeichnung bedarf es eines Antrages, wozu jedes AOTD-Mitglied berechtigt ist. Die antragstellende Person muss in leitender Funktion an einer herausragenden Klinik mit unfallchirurgischen Schwerpunkt tätig sein. Sie muss Mitglied der Akademie der AOTD sein. Pro Klinik ist nur eine Auszeichnung als „Leitender AOTrauma-Chirurg“ möglich.

Voraussetzung für die Zuerkennung der Auszeichnung ist die Erfüllung der vom Erweiterten Präsidium hierzu beschlossenen Kriterien.

Die Kriterien und das Antragsformular ist über die Geschäftsstelle der AOTD zu erhalten.

AOTrauma Deutschland

Herrn George Clay

E-Mail: g.clay@aodeutschland.de

Die nächste geplante Verleihung findet während des DKOU 2020 im Oktober statt. Die Deadline für die Einreichung des Antrages ist der 31. August 2020. 